\title{
Apport de la Technique ICP-MS à l'analyse toxicologique : la spéciation des métaux
}

\section{ICP-MS technique contribution to toxicological analysis : Metal speciation}

Hugues PAUCOT*, Hervé GARRAUD

UT2A, Hélioparc Pau Pyrénées, 2, av. du Président Angot 64053 PAU cedex 9.

*Auteur à qui adresser la correspondance : Hugues PAUCOT, UT2A, Hélioparc

Pau Pyrénées, 2 av. du Président Angot, 64053 Pau CEDEX 9, France Tél : 05.59.80.68.91 - Fax : 05.59.80.63.67 - E-mail : hugues.paucot@univ-pau.fr

(Reçu le 2 mars 2007 ; accepté après modifications le 21 mars 2007)

\section{RÉSUMÉ}

La détermination des états physico-chimiques d'un élément, que l'on appelle la spéciation d'un élément, constitue une source d'intérêt croissant au cours de ces dernières années. L'amélioration de la compréhension des processus physicochimiques conduisant à l'assimilation d'un élément, ou la mise en évidence de sa toxicité a entraîné une multiplication des développements analytiques en vue de l'analyse des différentes espèces. Ce type d'analyse comporte, tout d'abord, une phase de séparation réalisée par chromatographie liquide haute performance, chromatographie en phase gazeuse ou encore électrophorèse capillaire. Une fois cette séparation réalisée, la détermination des concentrations est réalisée par un système de détection. A ce jour, l'ICP-MS, du fait de sa sensibilité, de sa multi-élémentarité et de sa rapidité constitue la technique la plus utilisée à cette fin. L'objet de cet article est donc de faire le point sur les possibilités offertes par l'ICP-MS utilisé en couplage avec un système de séparation chromatographique, dans l'analyse de la spéciation des éléments traces dans les matrices biologiques.

\section{MOTS-CLÉS}

Spéciation, ICP-MS, chromatographie liquide hauteperformance, chromatographie en phase gazeuse, électrophorèse capillaire, ablation laser.

\section{SUMMARY}

Determination of the physico-chemical species of an element, called speciation analysis, has constituted an increasing source of interest during the last years. The improvement of the understanding of the physico-chemical processes driving to the assimilation of an element, or to the display of the toxicity has involved a multiplication of the analytical developments regarding the analysis of the various species.

This kind of analysis comprises, at first, a separation realized by High Performance Liquid Chromatography, Gas chromatography or Capillary Electrophoresis. Once this separation made, the determination of the concentrations is realized by a detector. ICP-MS is actually the most used technique for this goal due to its sensitivity, multi-elementarity and speed of analysis.

The goal of this publication is to strike the balance on the possibilities offered by ICP-MS used in hyphenation with a system of chromatographic separation, in the frame of speciation analyses of trace elements in biological matrices.

\section{KEY-WORDS}

Speciation, icp-ms, high performance liquid chromatography, gas chromatography, capillary electrophoresis, laser ablation. 


\section{Introduction}

La prise de conscience du rôle fondamental que joue la forme chimique d'un élément trace sur sa toxicité, sa biodisponibilité et sa mobilité dans les différents compartimentsdel'environnement, alargement progressé depuis une dizaine d'années. Par voie de conséquence, elle a aussi fortement stimulé le développement d'outils analytiques permettant l'étude des différentes formes sous lesquelles peuvent se trouver présents les éléments traces, ce que l'on appelle l'analyse de la spéciation d'un élément.L'enjeu est donc d'identifier et de quantifier les espèces chimiques, souvent présentes à des concentrations infimes dans des matrices très variées et plus ou moins complexes. Les analyses de spéciation trouvent donc un intérêt dans les domaines agroalimentaires ou environnementaux, par exemple, ou encore, en hygiène industrielle. Depuis quelques années, ce type d'études s'est également étendu aux divers milieux de la biologie, et en particulier de la biologie clinique $(1,2)$. A titre d'exemple, l'examen du devenir d'un médicament comportant un atome métallique pourrait en effet permettre d'en modifier la forme pour en optimiser l'efficacité. De même, la connaissance de l'évolution et du devenir d'une molécule organométallique toxique dans l'organisme humain permettrait d'en déduire, après coup, des informations quant au mode d'assimilation par la victime d'une intoxication, par exemple.

On distingue divers types de formes chimiques :

- Les formes dites rédox qui permettent de mettre en évidence les espèces ayant des états d'oxydo-réduction différents, tels que $\mathrm{As}^{\mathrm{III}}$ et $\mathrm{As}^{\mathrm{V}}, \mathrm{Se}^{\mathrm{IV}}$ ou ${ }^{\mathrm{VI}}$.

- les molécules organo-métalliques, telles que le tributylétain toxique utilisé dans les peintures antisalissures des navires, infiniment plus toxique que l'étain lui-même.

- les molécules d'origine biologique et de poids moléculaires supérieurs à 2000 daltons, dites macromolécules, telles que les arsénosucres, métalloprotéines, etc.

Quel que soit l'intérêt de cet aspect des choses, la réalisation d'analyse de spéciation ne s'improvise cependant pas. Elle nécessite en effet, au minimum, un système chromatographique séparateur pour pouvoir isoler les différentes espèces, et un détecteur pour les mettre en évidence et, le cas échéant, les quantifier. Parmi les différents détecteurs disponibles actuellement sur le marché, l'ICP-MS (Inductively Coupled Plasma - Mass Spectrometry) - association entre un plasma à couplage inductif et un spectromètre de masse - offre de nombreux avantages. Sa sensibilité, sa capacité multiélémentaire et la possibilité qu'il offre de réaliser des mesures isotopiques lui permettent d'entrer de plus en plus dans les laboratoires, et en particulier de remplacer les appareils d'absorption atomique électrothermique dans de nombreux laboratoires dont ceux de biologie clinique.

Du point de vue des analyses de spéciation, plusieurs constructeurs proposent des systèmes conçus pour une efficacité et une automatisation maximales. En effet, le système chromatographique est généralement muni d'un passeur automatique d'échantillons et le logiciel permet de déclencher automatiquement l'acquisition par l'ICP-MS. De plus, le logiciel de traitement des signaux transitoires générés par la technique est souvent complètement intégré au logiciel de l'ICPMS (pour une description plus complète des systèmes commercialement disponibles, nous vous renvoyons à la référence 3 ).

L'objet de cet article est donc de faire le point sur les possibilités offertes par l'ICP-MS utilisé en couplage avec un système de séparation chromatographique, dans l'analyse de la spéciation des éléments traces dans les matrices biologiques.

\section{Matériel et méthodes}

L'ICP-MS est constitué de l'association entre un plasma d'argon induit par couplage et un spectromètre de masse. Le rôle du plasma est de désolvater, dissocier et atomiser l'échantillon. Le spectromètre de masse aura pour tâche de séparer les différents ions présents et de sélectionner spécifiquement l'analyte afin de l'envoyer sur le détecteur. Plusieurs ouvrages ont décrit en détail les principes de fonctionnement de ce type d'appareil $(4,5)$.

L'utilisation de ce genre d'instrument dans le cadre des analyses de biologie clinique remonte à plus de 15 ans (6) et le nombre de publications se référant à son usage pour ce type d'applications ne cesse d'augmenter comme en témoignent les passages en revue réalisés régulièrement (7), ainsi que le nombre d'ICP-MS acquis par de nombreux hôpitaux français ou par plusieurs laboratoires d'analyses médicales privés. Certaines publications constituent d'ailleurs de véritables méthodes analytiques (8-10), preuves, s'il en est besoin, que l'ICP-MS est devenu un véritable outil d'analyse de routine pour de nombreux laboratoires de biologie clinique.

En ce qui concerne les analyses de spéciation, elles peuvent se subdiviser en plusieurs étapes, toutes aussi importantes les unes que les autres.

L'échantillonnage se devra, avant tout, de préserver la spéciation de l'analyte et d'éviter toutes formes de contamination. Il est à noter que plus l'espèce chimique est complexe, plus le risque d'une contamination est limité. Le problème qui se pose alors est de conserver correctement l'échantillon de telle sorte que les 
différentes espèces présentes ne soient pas modifiées par la lumière, la température, le temps et que la spéciation n'en soit dès lors pas affectée. Suit généralement une étape d'extraction, fonction de la nature de l'échantillon. Là encore, la contrainte majeure consiste à préserver la distribution de l'élément entre les différentes espèces mais aussi, à obtenir un rendement d'extraction suffisamment significatif (soit supérieur à $80 \%$ ). Une des techniques utilisées de plus en plus fréquemment pour corriger les modifications éventuelles qui peuvent se produire au cours de l'extraction fait appel à la dilution isotopique. Cette méthode est couramment utilisée dans le cas de l'analyse des espèces du mercure et de l'étain dans les tissus biologiques par GC-ICP-MS.

Différentes techniques séparatives peuvent être utilisées telles que la Chromatographie Liquide à Haute Performance (HPLC), la chromatographie en phase gazeuse (GC) ou l'électrophorèse capillaire (EC). La première reste, à ce jour, la plus usitée en raison de la simplicité de sa mise en œuvre.

Le choix entre ces différentes techniques passe avant tout par la nature des espèces que l'on souhaite analyser, en particulier la volatilité, ainsi que par la quantité d'échantillon dont on dispose.

Le dernier point important dont il convient de se préoccuper au cours des analyses de spéciation est la validation de la méthode. Diverses techniques sont envisageables telle que le dopage de l'échantillon et l'étude du taux de recouvrement. Une autre technique indiscutable permettant d'estimer le rendement d'extraction et la bonne conservation des espèces fait appel à l'utilisation de matériaux de référence certifiés (CRM). Malheureusement, le nombre de CRM disponibles commercialement en analyse de spéciation est encore limité comme le montre le tableau I, en particulier dans les matrices de biologie clinique (11).

Les développements de ces techniques d'analyse de spéciation dans le cadre des échantillons biologiques ont connu eux aussi un essor important comme en témoigne le nombre toujours croissant de publications (7).

\section{Le couplage HPLC - ICP-MS}

Le couplage entre un système de séparation par chromatographie en phase liquide haute performance (HPLC) et une détection par ICP-MS (figure 1) est certainement l'un des plus aisés à mettre en œuvre, en raison de la simplicité de l'interface. En effet, le couplage est réalisé en connectant simplement la sortie de la colonne chromatographique avec l'entrée du nébuliseur. Cette opération est rendue possible du fait de la compatibilité entre le débit de sortie de la colonne chromatographique et le débit d'entrée d'un nébuliseur pneumatique classique (approximativement $1 \mathrm{~mL} / \mathrm{min}$ ). De plus, il est possible d'utiliser plusieurs mécanismes
Tableau I : Liste des matériaux de référence certifiés dédiés à la spéciation. Les CRM entre guillemets ne sont pas certifiés.

\begin{tabular}{|c|c|}
\hline Paramètres certifiés & Matrices \\
\hline $\mathrm{Cr}(\mathrm{VI})$ & $\begin{array}{c}\text { Bichromate de potassium (SRM 136e), } \\
\text { Poussières de soudure sur filtre (BCR-545), } \\
\text { solution étalon (SRM 2109) }\end{array}$ \\
\hline $\mathrm{Cr}(\mathrm{III}), \mathrm{Cr}(\mathrm{VI})$ & Solution lyophilisée (BCR-544), \\
\hline $\mathrm{Cr}(\mathrm{III})$ & Solution étalon (SRM 2108) \\
\hline $\begin{array}{l}\mathrm{Hg} \text { total et Methyl Hg } \\
(\mathrm{MeHg})\end{array}$ & $\begin{array}{l}\text { Sédiment (ERM-CC580), Sédiment estuarien } \\
\text { (IAEA-405), Sédiment marin (IAEA-433), Tis } \\
\text { sus de moule (SRM 1974b, SRM 2976, SRM } \\
\text { 2977), Tissu d'huître (SRM 1566b), Tissu de } \\
\text { Thon (BCR-463 et ERM-CE464), Pancréas } \\
\text { de Homard (TORT-2), Roussette (DORM-2, } \\
\text { « BCR-422», « DOLT-3», NMIJ 7402-a), } \\
\text { Poisson d'eau douce (SRM 1946), Poisson } \\
\text { (IAEA-407), Cheveux humains (IAEA 085 et } \\
\text { 086, NIES 13), sang de bœuf (SRM 966) } \\
\end{array}$ \\
\hline $\begin{array}{l}\text { Butylétains } \\
\text { (TBT, DBT) }\end{array}$ & $\begin{array}{c}\text { Sédiments marin (HIPA-1, SOPH-1, PACS-2, } \\
\text { NMIJ 7306-a et NIES 12), côtiers (BCR-462), } \\
\text { d'eaux douces (BCR-646), tissus de moule } \\
\text { (ERM-CE477), de poissons (NIES 11) }\end{array}$ \\
\hline $\begin{array}{l}\text { Arséno Bétaïne } \\
\text { (AsB) }\end{array}$ & $\begin{array}{c}\text { Solution étalon (BCR-626 et NMIJ 7901-a), } \\
\text { Roussette (NMIJ 7402-a) }\end{array}$ \\
\hline $\begin{array}{l}\text { ArsénoBétaïne, } \\
\text { DiMéthyl Arsenic }\end{array}$ & Thon (BCR-627), Urine (NIES 18) \\
\hline $\begin{array}{l}\text { ArsénoBétaïne (AsB), } \\
\text { TetraMéthylArsinique }\end{array}$ & Roussette (DORM-2) \\
\hline SelenoMéthionine & Levure enrichie (SELM-1) \\
\hline Triméthylplomb & Poussières urbaines (BCR-605) \\
\hline TBT, DBT, MeHg, AsB & $\begin{array}{l}\text { Tissu d'huître («BCR-710») } \\
\text { en cours de certification }\end{array}$ \\
\hline
\end{tabular}

de séparation (e.g. échange d'ions, chromatographie de phase inverse avec ou sans appariement d'ions, exclusion stérique) qui permettent l'analyse de composés chimiques très différents comme les espèces redox, les composés organométalliques, les biomolécules, des métabolites de xénobiotiques. La principale limitation de ce type de couplage est le manque de compatibilité de la phase mobile indispensable à l'HPLC avec l'ICPMS. En effet, des teneurs trop élevées en sels ou en solvants organiques peuvent induire des perturbations au niveau du nébuliseur, du plasma et/ou des cônes.

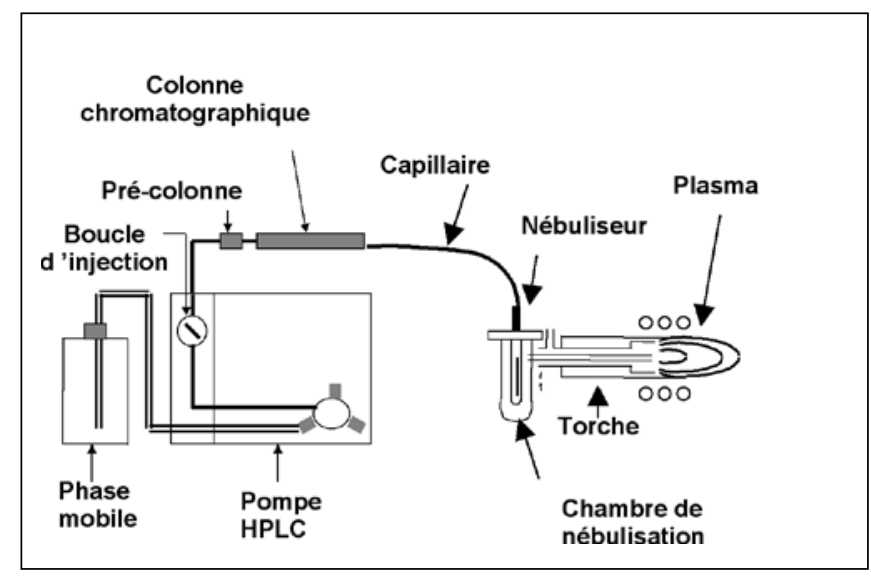

Figure 1 : Schéma général du couplage HPLC-ICP-MS. 
Dans le cas des sels, les problèmes peuvent être minimisés en rinçant périodiquement le système et en maintenant une teneur en sels inférieure à $2 \%$. Dans le cas de solvants, la concentration dans l'éluant ne doit idéalement pas dépasser $3 \%$. Il peut aussi être nécessaire d'insérer une unité de désolvatation, de refroidir la chambre de nébulisation, d'ajouter de l'oxygène au gaz de nébulisation ou de faire une dilution post-colonne. De plus, s'il est souvent préférable de travailler en mode gradient lors du processus de séparation chromatographique, la modification de la matrice peut influencer les conditions du plasma et conduire à une dérive du signal. Le tableau II synthétise les différentes conditions qui peuvent être mises en ouvre pour ce type de séparation.

Tableau II : Conditions opératoires mises en æuvre pour l'analyse par couplage HPLC - ICP-MS (13).

\begin{tabular}{|l|c|}
\hline Type de séparation & $\begin{array}{c}\text { Echange d'ions, exclusion stérique, phase } \\
\text { inverse avec ou sans appariement d'ion }\end{array}$ \\
\hline Espèces séparées & $\begin{array}{c}\text { Espèces rédox, composés organo-métalliques, } \\
\text { biomolécules }\end{array}$ \\
\hline Débit (ml/min) & $\begin{array}{r}80 \mu \mathrm{ll} / \mathrm{min} \text { avec des micro-colonnes } \\
0,75 \mathrm{à} 1,5 \mathrm{ml} / \mathrm{min} \text { avec des colonnes normales }\end{array}$ \\
\hline Pression (bar) & 60 à 110 \\
\hline Elution & Gradient ou isocratique \\
\hline Volume injecté $(\mu \mathrm{l})$ & 50 à 100 \\
\hline Résolution & 100 à 5000 plateaux théoriques \\
\hline Durée de l'analyse $(\mathrm{min})$ & 2 à 20 \\
\hline
\end{tabular}

\section{Le couplage GC - ICP-MS}

Le couplage entre un chromatographe en phase gazeuse (GC) et un ICP-MS est composé de trois parties distinctes : le four de chromatographie équipé d'une colonne capillaire apolaire, une interface, et l'ICPMS (figure 2). Dans tous les cas, les espèces volatiles, préalablement solubilisées dans un solvant organique au cours de la préparation de l'échantillon, sont introduites en tête de colonne par un port d'injection soit de manière manuelle, soit de manière automatique. Elle sont alors séparées et transportées par le gaz vecteur jusqu'au plasma de l'ICP-MS. L'interface qui assure

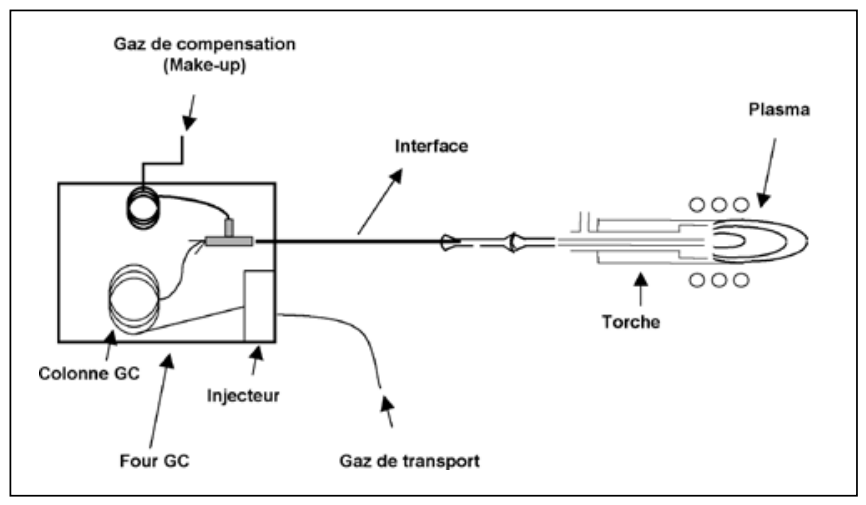

Figure 2 : Schéma général du couplage $G C-I C P-M S$. la connexion entre le four et le plasma, doit maintenir sur toute sa longueur une température proche de celle du four afin d'éviter la condensation des espèces, et avoir un faible volume mort pour ne pas dégrader la résolution chromatographique du couplage. Le débit du gaz vecteur étant insuffisant pour assurer l'introduction des espèces au cœur du plasma, un gaz additionnel (en général de l'argon à $0,3-1 \mathrm{~L} / \mathrm{min}$ ) est ajouté à l'entrée de l'interface. Un apport d'oxygène peut parfois aussi être nécessaire pour favoriser la combustion du carbone issu du solvant organique dans le plasma. D'un point de vue commercial, plusieurs constructeurs proposent actuellement ce genre d'interface (3). La préparation de l'échantillon est une étape décisive dans le protocole analytique et joue ici un double rôle. Tout d'abord, il s'agit d'extraire quantitativement les espèces à analyser de la matrice. Une extraction sous champ micro-ondes à faible puissance en milieu acide ou basique selon la situation permet de réaliser cette opération. Puis, dans la grande majorité des cas, il sera nécessaire de transformer les analytes (souvent présents sous forme ionique dans l'environnement) en espèces volatiles, pouvant ainsi être séparées par chromatographie en phase gazeuse. On réalise cela par une réaction de dérivation consistant à greffer un ou plusieurs groupements alkyls (ethyl, propyl ou butyl) à l'analyte, de manière à obtenir un composé totalement substitué et volatil que l'on récupère dans un solvant organique. Le tableau III synthétise les différentes conditions mises en œuvre pour ce type de séparation.

Tableau III : Conditions opératoires mises en æuvre pour l'analyse par couplage $G C-I C P-M S$.

\begin{tabular}{|l|c|}
\hline Type de séparation & Colonne polaire ou apolaire \\
\hline Gaz vecteur & He \\
\hline Débit $(\mathrm{ml} / \mathrm{min})$ & $1-100$ \\
\hline Volume injecté $(\mu \mathrm{l})$ & $1-5$ \\
\hline Résolution & 1000 à 10000 plateaux théoriques \\
\hline Température de l'interface $\left({ }^{\circ} \mathrm{C}\right)$ & $200-300$ \\
\hline Durée de l'analyse $(\mathrm{min})$ & $5-60$ \\
\hline
\end{tabular}

\section{Apport de la dilution isotopique :}

La dilution isotopique est une méthode dite «primaire » qui permet de corriger les artéfacts qui se produisent lors de la préparation des échantillons. Elle consiste à ajouter un isotope marqué (Exemples : ${ }^{111} \mathrm{Cd}$, Méthyl- ${ }^{202} \mathrm{Hg}$ ) lors de la préparation des échantillons. La molécule ajoutée se comportera de façon identique aux espèces naturelles. Ainsi, il devient alors possible de connaître avec précision les rendements d'extraction liquide-liquide, des réactions de dérivation, ou encore de corriger les effets de matrice et les dérives des signaux. 
Les concentrations des espèces chimiques recherchées sont calculées par les rapports isotopiques entre espèces naturelles et enrichies. Une équation, définie dans le logiciel de l'appareillage ou par un tableur, permet une exploitation rapide de cette méthode. La méthode dite des double ajouts (Deux espèces chimiques et deux isotopes d'un même élément) permet, quant à elle, de quantifier et de corriger les réactions de conversion interespèces (Exemples : $\mathrm{Cr}$ (III) et $\mathrm{Cr}$ (VI) ou $\mathrm{Hg}^{2+}$, Methyl$\mathrm{Hg}$ ). La dilution isotopique permet ainsi d'améliorer sensiblement la fidélité et la justesse de l'analyse.

\section{Le couplage CE-ICP-MS}

L'importance de l'électrophorèse capillaire (EC) a beaucoup augmenté ces dernières années du fait de sa complémentarité avec l'HPLC mais aussi d'un pouvoir de résolution extrêmement important (13). Le principe de cette séparation est basé sur les différentes propriétés migratoires d'espèces chargées le long d'un tube capillaire placé dans un champ électrique constant (Figure 3). Le capillaire est rempli avec un tampon conducteur adéquat et le champ électrique est établi en appliquant un gradient de tension via des électrodes en platine aux deux extrémités du capillaire flexible. Les capillaires sont réalisés en silice fondue et contiennent en surface des groupements silanol $(\mathrm{SiOH})$. Typiquement, les capillaires ont un diamètre intérieur de 20 à $100 \mu \mathrm{m}$ et une longueur de 20 à $100 \mathrm{~cm}$. Les volumes injectés sont de quelques nanolitres.

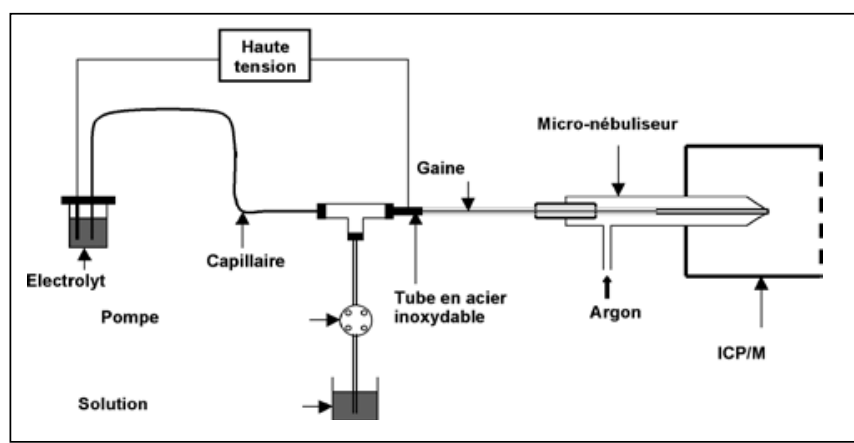

Figure 3 : Schéma général du couplage EC-ICP-MS.

La connexion avec un ICP-MS présente un certain nombre de difficultés et actuellement, une seule interface commerciale est disponible (14). Dans tous les cas, l'interface en question doit s'adapter au faible débit de l'EC par l'utilisation d'un liquide d'appoint, du fait que la plupart des nébuliseurs pour ICP-MS sont étudiés pour fonctionner à des débits de l'ordre de dizaines de $\mu \mathrm{L}$ ou de $\mathrm{mL} / \mathrm{min}$. Un courant électrique stable est crucial pour des séparations reproductibles. Enfin, le volume mort de l'interface, qui pourrait causer des élargissements de pics, doit être minimisé au maximum. Le tableau IV synthétise les différentes conditions mises en œuvre pour ce type d'analyses.
Tableau IV : Conditions opératoires mises en æuvre pour l'analyse par couplage EC - ICP-MS (13).

\begin{tabular}{|l|c|}
\hline Type de colonne & $\begin{array}{c}\text { Silice fondue avec groupements silanol } \\
(\mathrm{SiOH})\end{array}$ \\
\hline Espèces séparées & $\begin{array}{c}\text { Molécules anioniques, cationiques et neutres } \\
\text { de poids moléculaire faible à élevé }\end{array}$ \\
\hline Gradient de tension $(\mathrm{kV})$ & 10 à 30 \\
\hline Débit $(\mathrm{nl} / \mathrm{min})$ & $<150$ \\
\hline Volume injecté $(\mathrm{nl})$ & 2 à 20 \\
\hline Résolution & 500000 à 1000000 plateaux théoriques \\
\hline Durée de l'analyse $(\mathrm{sec})$ & $<500$ \\
\hline
\end{tabular}

\section{Le couplage ablation laser - ICP-MS}

L'analyse par ablation laser - ICP-MS consiste à transférer, sous l'effet d'impulsions laser (généralement de quelques nano, voire femtosecondes), une fraction de l'échantillon sous forme de fines particules ou de vapeurs dans le plasma (Figure 4). La taille des particules formées est très dépendante des propriétés du matériau et de la nature du faisceau laser, allant de quelques nanomètres (avec les lasers les plus performants) à quelques micromètres. Pour ce faire, l'échantillon est placé dans une cellule d'ablation balayée par un flux de gaz vecteur ( $\mathrm{Ar}, \mathrm{He}$, etc) transportant la matière ablatée jusqu'à l'ICP-MS. Un système optique permet de focaliser le faisceau laser sur la surface à analyser et de régler la taille du faisceau (dont dépend la quantité de matière ablatée).

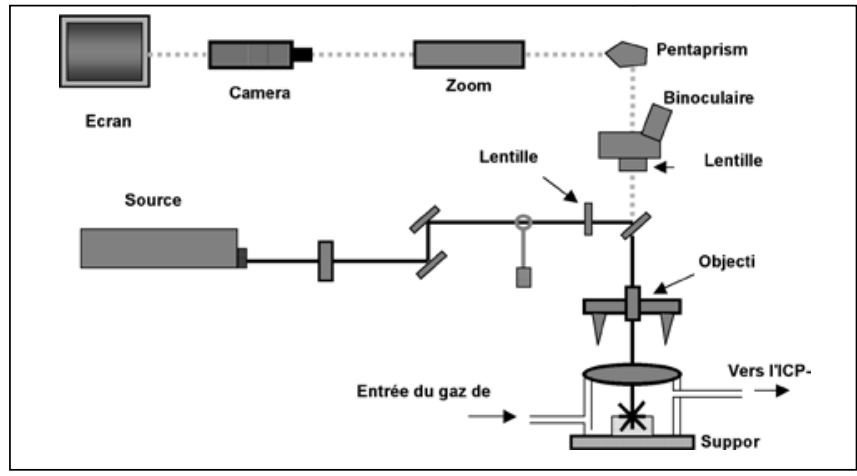

Figure 4 : Schéma général d'un système d'ablation laser.

L'ablation laser ne nécessitant pas de mise en solution préalable de l'échantillon, elle se révèle plus facile à mettre en œuvre, plus rapide et présente moins de risques de contamination que les analyses conventionnelles par voie humide. Elle permet en outre de réaliser des analyses microscopiques (typiquement 5 à $200 \mu \mathrm{m}$ ) pour cartographier la composition élémentaire de la surface de l'échantillon par exemple, ou bien pour déterminer sa composition élémentaire en profondeur. Ainsi, cette technique est devenue un outil intéressant pour l'identification de métalloprotéines séparées sur gel d'électrophorèse, avant leur analyse par ICP-MS (15- 
16) ou leur caractérisation par spectrométrie de masse ESI/MS/MS. Les niveaux de détection pour le sélénium, peuvent être inférieurs à $50 \mathrm{ppt}$, ce qui contribue à l'identification de nouvelles protéines séléniées.

\section{Exemples d'application}

Comme il est, de loin, le plus aisé à mettre en œuvre, le couplage HPLC - ICP-MS a été utilisé dans le cadre d'analyse biologique par de nombreux auteurs pour la spéciation de l'aluminium (17), de l'arsenic (18-24), du platine (25-26), du sélénium (27-29), de l'antimoine (30), du cadmium (31), du chrome (32) ou du manganèse (33), par exemple.

Seules quelques applications sont présentées ici. Elles visent à montrer les possibilités de la technique en analyse toxicologique dans l'analyse d'espèces redox, des composés organométalliques, des biomolécules, des métabolites de xénobiotiques.

Ainsi la spéciation de l'Al dans des cellules de neuroblastome exposées au lactate d'aluminium, a pu, par exemple, être réalisée (17) par chromatographie d'exclusion stérique. $100 \mu \mathrm{L}$ d'échantillon ont été injectés à une vitesse de $0,75 \mathrm{~mL} / \mathrm{min}$. Cette application a permis de mettre en évidence une nouvelle molécule de faible poids moléculaire.

La spéciation de l'arsenic dans des échantillons d'urine (figure 5) ou de cheveux (figure 6) montre également quelques exemples des possibilités de la technique. Dans ce cas, les colonnes utilisées réalisent la séparation par échange de cations ou d'anions (34).

Elle a également été appliquée à la spéciation de l'arsenic dans un tissu d'huître (35). En effet, si l'arsenic inorganique est bien connu pour sa grande toxicité, certaines formes organiques, comme l'arsénobétaïne, composé arsénié majoritaire dans les poissons et fruits de mer, sont moins toxiques pour l'homme que l'aspirine. Aussi, la connaissance précise de la spéciation de cet élément permet d'apporter plus de discernement dans la qualité alimentaire ou pernicieuse du produit en question (36).

Une autre application est l'étude du suivi d'un médicament et de ses métabolites dans l'organisme humain. Ainsi, Vacchina et al (37) ont analysé les résidus d'un médicament à base de platine par couplage HPLC (échange de cations) et ICP-MS. La figure 7 montre le chromatogramme obtenu. On y distingue le résidu de la molécule d'origine ainsi que les différents métabolites. Dans certains cas, il peut s'avérer nécessaire d'enchaîner les techniques de séparation, afin d'optimiser la purification des différentes espèces présentes. Ainsi, dans l'exemple suivant, la méthode développée (38) pour déterminer précisément les acides aminés séléniés dans du sérum humain, fait tout d'abord appel à la

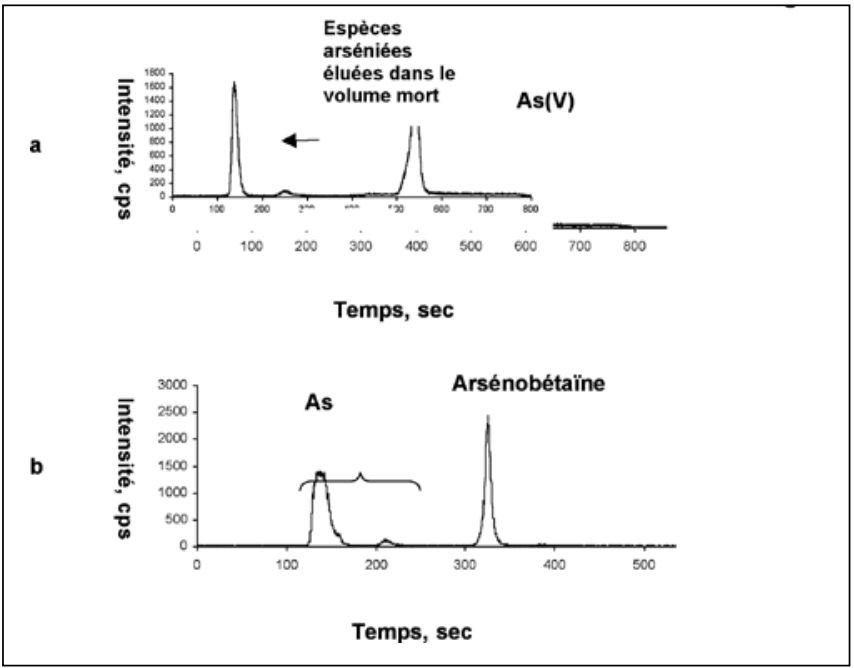

Figure 5 : Chromatogrammes d'un échantillon d'urine sur colonne échangeuse d'anions et de cations (34). Conditions opératoires (a) : Colonne : Hamilton PRPX-100 ; phase mobile : 0 à 4 min : Tampon phosphate $5 \mathrm{mM}, \mathrm{pH}=4,7 ; 4$ à $10 \mathrm{~min}$ : Tampon phosphate $30 \mathrm{mM}, \mathrm{pH}=8 ; 10$ à $20 \mathrm{~min}$ : Tampon phosphate $5 \mathrm{mM}, \mathrm{pH}=4,7$; débit : $1 \mathrm{~mL} / \mathrm{min}$; volume injecté : $100 \mu \mathrm{L} .(\mathrm{b})$ : Colonne : Hamilton PRPX-200 ; phase mobile : 0 à $1 \mathrm{~min}: 90 \% \mathrm{~A}, 10 \% \mathrm{~B} ; 1$ à $2 \mathrm{~min}$ : $40 \% \mathrm{~A}, 60 \% \mathrm{C} ; 2$ à $3,5 \mathrm{~min}: 20 \% \mathrm{~A}, 80 \% \mathrm{C} ; 3,5$ à $6 \mathrm{~min}: 20 \% \mathrm{~A}$, $80 \% \mathrm{~B} ; 6$ à $10 \mathrm{~min}: 100 \% \mathrm{~A} ; 10$ à $20 \mathrm{~min}: 90 \% \mathrm{~A}, 10 \% \mathrm{~B} ;$ où $\mathrm{A}$ $: \mathrm{KNO}_{3} 25 \mathrm{mM}, \mathrm{pH}=6 ; \mathrm{B}: \mathrm{HNO}_{3} 25 \mathrm{mM}, \mathrm{pH}=1,6 ; \mathrm{C}: \mathrm{NaOH}$ $25 \mathrm{mM}, \mathrm{pH}=12,4$; débit : $1 \mathrm{~mL} / \mathrm{min}$; volume injecté : $100 \mu \mathrm{L}$.

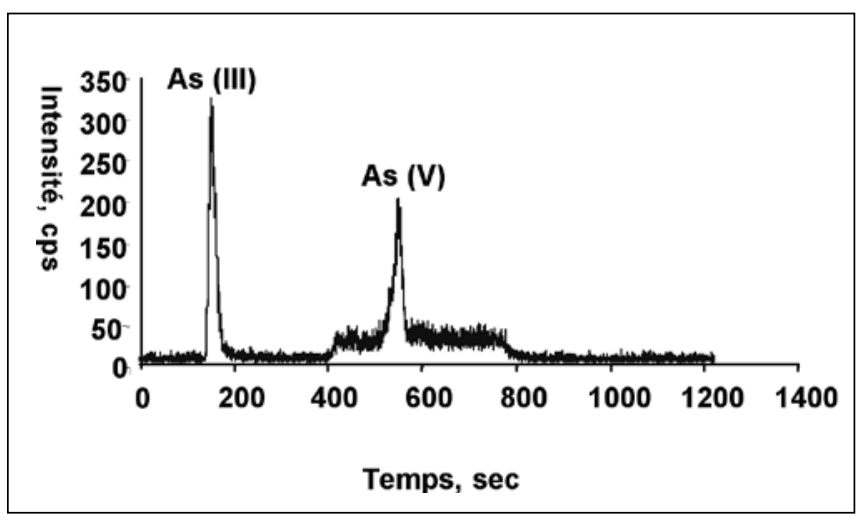

Figure 6 : Chromatogramme d'un échantillon d'extrait aqueux de cheveux sur colonne échangeuse d'anions (34). Conditions opératoires : Colonne : Hamilton PRPX-100; phase mobile : 0 à $4 \mathrm{~min}:$ Tampon phosphate $5 \mathrm{mM}, \mathrm{pH}=4,7 ; 4$ à $10 \mathrm{~min}:$ Tampon phosphate $30 \mathrm{mM}, \mathrm{pH}=8 ; 10$ à $20 \mathrm{~min}$ : Tampon phosphate $5 \mathrm{mM}$, $p H=4,7$; débit : $1 \mathrm{~mL} / \mathrm{min}$; volume injecté : $100 \mu \mathrm{L}$.

chromatographie par exclusion stérique pour isoler la fraction contenant les acides aminés séléniés, puis à une séparation de la sélénométhionine et de la sélénocystéîne carboxyméthylée par HPLC capillaire. Dans ce cas, la quantification a été réalisée par dilution isotopique d'un ajout de sélénométhionine marquée au ${ }^{77} \mathrm{Se}$ et la détermination du rapport ${ }^{77} \mathrm{Se} /{ }^{80} \mathrm{Se}$ sur le pic de sélénométhionine. La figure 8 illustre les possibilités de cet enchaînement de techniques séparatives.

Le couplage HPLC-ICP-MS, en faisant appel à la chromatographie en phase inverse avec appariement 


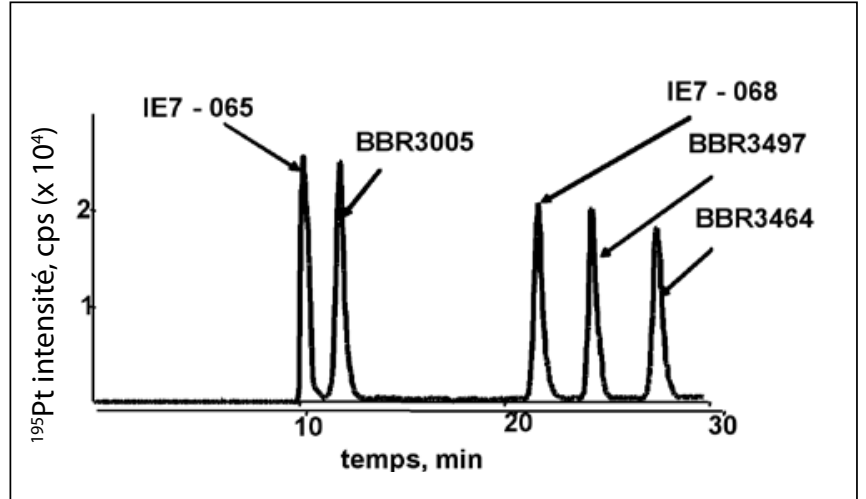

Figure 7 : Chromatogramme obtenus par HPLC en échange de cations - ICP-MS d'un médicament (BBR 3464) à base de platine et de ses métabolites (arbitrairement dénommés) (37). Conditions opératoires : Colonne Supelcosil LC-SCX (250 x 4,6 mm d.i.) ; phase mobile : de 20 à $200 \mathrm{mM}$ de Pyridine $(\mathrm{pH}=3)$ en $30 \mathrm{mn}$; débit : $1 \mathrm{~mL} / \mathrm{min}$; volume injecté : $100 \mu \mathrm{L}$.

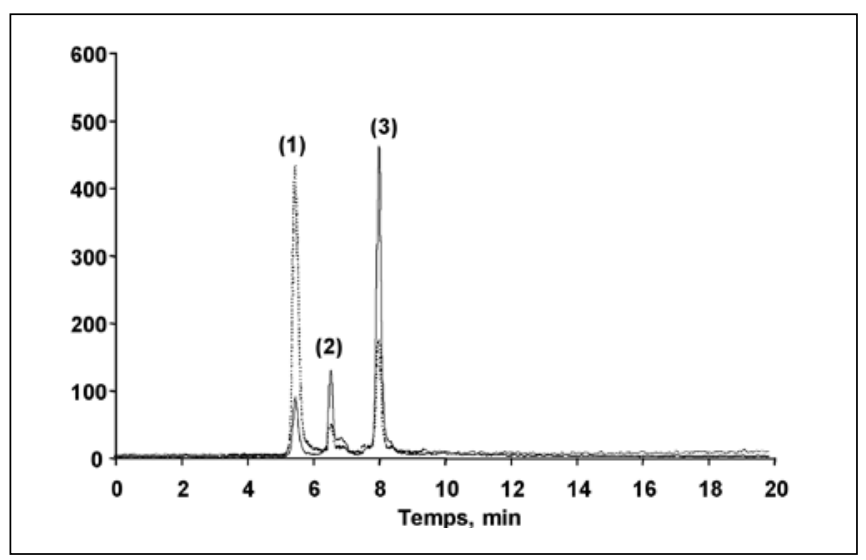

Figure 8 : Chromatogramme HPLC-capillaire en phase inverse - ICP-MS de la fraction d'acides aminés séléniés collectée en exclusion de taille (38). Identification des pics : (1) Sélénocystéine carboxyméthylée, (2) oxyde de sélénométhionine, (3) Sélénométhionine. Ligne continue : ${ }^{77} \mathrm{Se}$, trait interrompu : ${ }^{80} \mathrm{Se}$. Noter la différence de rapport isotopique ${ }^{77} \mathrm{Se} /{ }^{80} \mathrm{Se}$ dans les pics de selenocysteine et de sélénométhionine du fait de l'ajout de sélénométhionine marquée au ${ }^{77} \mathrm{Se}$. Conditions opératoires : Colonne Hypersil $C^{18}$ BDS (300 $\mu \mathrm{m}$ d.i. $x 15 \mathrm{~cm}, 3 \mu \mathrm{m}$, LC Packings); phase mobile : A, 5\% acétonitrile / 0,1\% TFA, B, 95\% acétonitrile / $0,1 \%$ TFA - 0 à $15 \mathrm{~min}: 10$ à $60 \%$ B (linéaire) - 15 à $20 \mathrm{~min}: 60 \%$; débit : $4 \mu \mathrm{L} / \mathrm{min}$; volume injecté : $200 \mathrm{~mL}$.

d'ions, permet également le dosage des espèces du mercure (mercure inorganique et méthylmercure), du plomb (e.g. plomb tétraéthyl des essences) et de l'étain (composés butylés et phénylés de l'étain). Toutefois, cette technique est généralement handicapée par l'obligation d'utiliser un ou des solvants organiques lors de la séparation chromatographique (on parle de phase inverse), ce qui se traduit par une difficulté accrue au niveau de la détection par ICP-MS. Si ce type d'analyse reste possible comme l'illustre la figure 9 pour de l'analyse de $\mathrm{Hg}$ et de Méthyl-Hg dans de l'urine dopée, la durée de l'analyse, le rendement quotidien en termes d'échantillons analysés et les limites de détection atteintes sont en général moins performants que par couplage GC-ICP-MS.

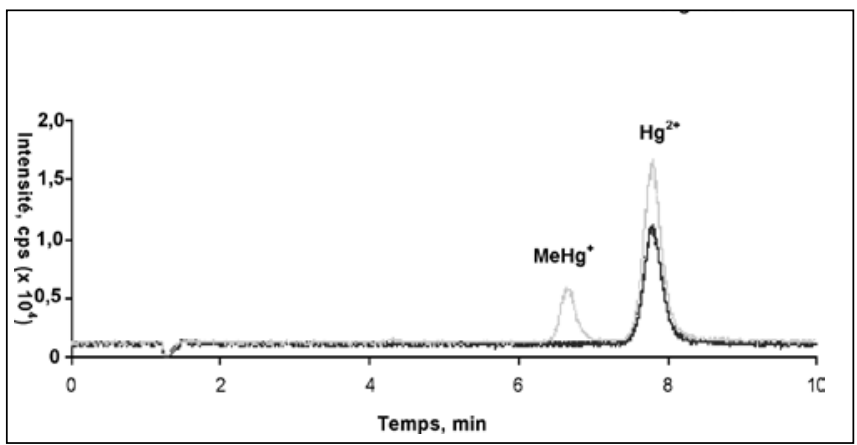

Figure 9 : Chromatogramme obtenu par couplage HPLC - ICP$\mathrm{MS}$ du Hg et du Méthyl-Hg dans une urine dopée (40). Conditions

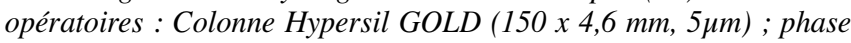
mobile : 60 mMacétate d'ammonium, 5\% méthanol, 2-mercaptoethanol 0,01\% ; débit : $1,5 \mathrm{~mL} / \mathrm{min}$; volume injecté : $100 \mu \mathrm{L}$.

De son côté, l'utilisation de l'électrophorèse capillaire couplée à un ICP-MS a surtout été utilisée pour la spéciation des métalloprotéines. Ainsi l'aluminium (17) et le sélénium $(27,28)$ ont vu leur spéciation réalisée par cette voie. Son application reste cependant encore très confidentielle du fait des difficultés de mise en œuvre.

En ce qui concerne le couplage GC-ICP-MS, son intérêt réside, entre autres, dans sa sensibilité et dans le fait qu'elle permet d'éviter la phase inverse en couplage HPLC-ICP-MS, lors de l'analyse des espèces du mercure par exemple. Parmi celles-ci, se trouve le Méthylmercure, dont la toxicité incite les institutions sanitaires à émettre des recommandations très précises quant à la consommation de produits de la mer (39). Ces développements ont fait l'objet d'une communication lors de la Winter Conference en 2006 (40). Toutefois, les difficultés liées à la matrice lors de l'étape de dérivation, indispensable lors du couplage GC-ICP-MS, rendent cette dernière technique encore difficilement applicable aux échantillons biologiques, du fait du mauvais taux de recouvrement.

Enfin, une application particulière est fondée sur l'utilisation de l'ablation laser et son usage pour l'identification de nouvelles protéines comportant un ou plusieurs atomes métalloïdiques tels que le sélénium par exemple. Cette étude est réalisée par l'ablation sur un gel électrophorétique, éventuellement bidimensionnel $(15,16)$, après séparation des différentes protéines en fonction de leur poids moléculaire. La figure 10 montre ainsi l'intérêt de l'ablation laser dans la démonstration de la présence de Se dans une protéine spécifique préalablement séparée par ICP-MS (41).

\section{Discussion}

A travers les exemples montrés ici, les potentialités de l'ICP-MS pour les analyses de spéciation dans les analyses toxicologiques apparaît assez clairement. Que ce soit pour l'étude de médicament où l'on voit tout 


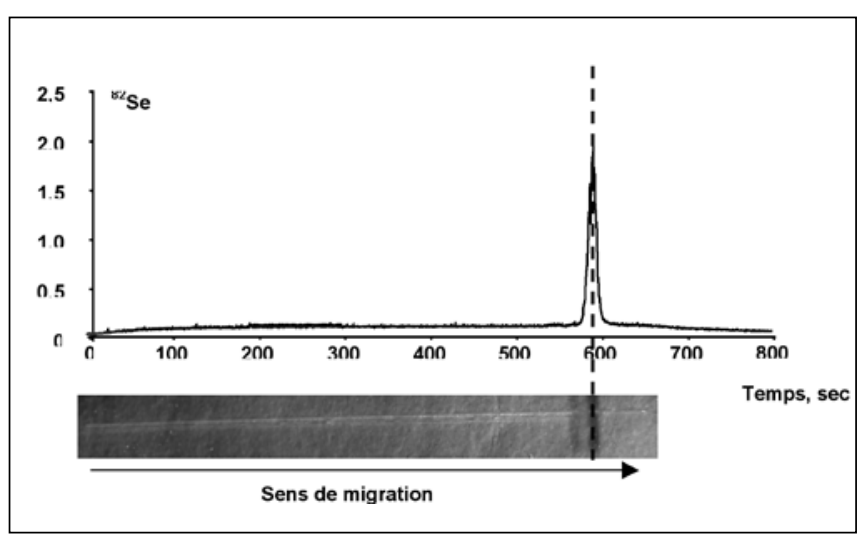

Figure 10 : Ablation laser-ICP-MS d'un Electrophérogramme 1-D $S D S$ de Se-Méthionine (41). Electrophorèse réalisée dans du Tris $25 \mathrm{mM}$ contenant 3,5 mM SDS et $192 \mathrm{ML}$ L-glycine à $180 \mathrm{~V}$ pendant $45 \mathrm{~min}$.

l'intérêt de l'étude du devenir de la molécule d' origine, et de ses éventuels excipients, ou pour l'analyse d'espèces particulières dans les fluides biologiques.

Deux restrictions importantes limitent toutefois encore le développement des analyses de spéciation : la stabilité des espèces entre le moment du prélèvement et l'analyse, d'une part, et la disponibilité de matériaux de référence spécifiques, d'autre part. Devant cette difficulté, nombre d'analystes s'orientent de plus en plus vers les matériaux de référence de laboratoire.

Une perspective encore plus ample dans le domaine est la collaboration entre différentes techniques analytiques (42). Ainsi, si des techniques comme les spectromètres de masse en tandem (MS-MS) ou temps de vol (TOF), couplés à une ionisation MALDI ou Electrospray (ESI) sont des outils extrêmement puissants pour l'étude d'espèces complexes dans le cadre d'étude de protéomique ou de génétique, leur capacité en terme de quantification est extrêmement limitée. A l'inverse, l'ICP-MS, une fois la fraction contenant l'espèce intéressante parfaitement isolée, sera à même de la quantifier, pour autant, bien sûr, que celleci contienne un ion métallique ou métallö̈de susceptible d'être analysé. Ainsi, par exemple, plusieurs études ont permis de purifier des métabolites contenant du Se, de les identifier par ESI-MS ou par nano-ESI-MS, puis de les quantifier par HPLC- ou CE- ICP-MS $(27,28)$. Parmi les études réalisées au laboratoire, certaines ont permis de développer nos connaissances sur la sélénométhionyl calmoduline (41).

\section{Conclusion}

L'intérêt de l'ICP-MS dans les analyses toxicologiques parait donc relativement important. En effet, hors du domaine biomédical à proprement parler, ses capacités analytiques (rendement, limites de quantification, etc.) ont permis à cette technique de s'imposer dans de nombreux laboratoires pour l'analyse de routine de diverses espèces. En environnement, son utilisation pour la spéciation de l'arsenic, du chrome du mercure est maintenant chose courante. En biologie clinique, les nombreux développements présentés ici montrent toutes les potentialités du système. De plus, favorisé par les améliorations techniques et encouragé par les efforts des constructeurs en vue d'augmenter l'automatisation, le développement de ce type d'analyse ne peut que continuer à croître, jusqu'à devenir partie intégrante des analyses dites de routine.

\section{Remerciements}

Les auteurs tiennent à remercier Fabienne Séby et Véronique Vacchina d'UT2A, Christophe Peycheran, Guillaume Ballihaut et Joanna Szpunar du LCABIE - CNRS UMR 5034, pour leur aide, leurs conseils et les informations fournies, ainsi que les sociétés Agilent Technologies et Thermo Elemental pour l'aide technique fournie qui a permis à l'ensemble de l'équipe d'obtenir les résultats présentés.

\section{Références}

1. Allain P. Dosage et spéciation des métaux dans les milieux biologiques : d'hier à aujourd'hui. Ann. Toxicol. Anal. $2001 ; 13$ : 167-8.

2. Anger J.P., Labat L., Lhermitte M. La biométhylation des métaux chez l'homme et dans l'environnement : succès ou échec sur le plan toxicologique. Ann. Toxicol. Anal. $2005 ; 17: 175-85$.

3. Paucot H. Etat de l'art et derniers développements en ICP-MS. Ann. Toxicol. Anal. 2007 ; 19 : 3-10.

4. Montaser A. Inductively Coupled Plasma Mass Spectrometry. Wiley-VCH, 1998.

5. Nelms S. ICP Mass Spectrometry Handbook. Blackwell Publishing, CRC Press, 2005.

6. Templeton D.M., Paudyn A., Baines A.D. Multielement analysis of biological samples by inductively coupled plasma-mass spectrometry. I. Preliminary considerations and analysis of rat liver and serum. Biol. Trace Elemen. Res. 1989 ; 22 : 17-33.

7. Taylor A., Branch S., Halls D., Patriarca M., Whaite M. Atomic spectroscopy update. Clinical and biological materials, foods and beverages. J. Anal. Atom. Spectrom. $2005 ; 20: 323-69$.

8. Bonnefoy C., Menudier A., Moesch C., Lachâtre G., Mermet J.-M. Validation of the determination of lead in whole blood by ICP-MS. J. Anal. Atom. Spectrom. $2002 ; 17: 1161-5$.

9. Goullé J.P., Mahieu L., Castermant J., Neveu N., Laine G., Nouveau M.P., Gehanne R., Bouige D., Lacroix C. Validation d'une technique de dosage multi-élémentaire des métaux par ICP-MS dans les milieux biologiques. Ann. Toxicol. Anal. $2003 ; 15$ : 271-80. Correctif dans Ann. Toxicol. Anal. 2004 ; 16 : 257-60. 
10. Goullé J.P., Mahieu L., Bonneau L., Laine G., Bouige D., Lacroix C. Validation d'une technique de dosage multiélémentaire des métaux et métalloïdes dans les cheveux par ICP-MS. Valeurs de référence chez 45 témoins. Ann. Toxicol. Anal. 2005 ; 17 : 97-103.

11. McSheehy S., Szpunar J., Morabito R., Quevauviller P. The speciation of arsenic in biological tissues and the certification of reference materials for quality control. Trends Anal. Chem. 2003 ; 22 :191-209.

12. Monperrus M., Krupp E., Amouroux D., Donard O., Rodriguez Martin-Doimeadios R.C. Potential and limits of speciated isotope-dilution analysis for metrology and assessing environmental reactivity. Trends Anal. Chem. $2004 ; 23$ : 261-72.

13. Krupp E., Seby F., Rodriguez R., Holliday A., Moldovan M., Köllensperger G., Hann S., Donard. O. Trace metal speciation with ICP-MS Detection in Nelms, S. ICP Mass Spectrometry Handbook. Blackwell Publishing, CRC Press, 2005 : 259-335.

14. Schaumlöffel D., Prange A. A new interface for combining capillary electrophoresis with inductively coupled plasma - mass spectrometry. Fresenius J. Anal. Chem. 1999 ; $364: 452-6$.

15. Chéry C., Günther D., Cornelis R., Vanhaecke F., Moens L. Detection of metals in proteins by means of polyacrylamide gel electrophoresis and laser ablationinductively coupled plasma-mass spectrometry : Application to selenium. Electrophoresis $2003 ; 24$ : 3305-13.

16. Becker J.S., Zoriy M., Becker J.S., Pickhardt C., Przybylski M. Determination of phosphorus and metals in human brain proteins after isolation by gel electrophoresis by laser ablation inductively coupled plasma source mass spectrometry. J. Anal. Atom. Spectrom. 2004 ; 19 : 14952.

17. Polec-Pawlak K., Zambenedetti P., Szpunar J., Lobinski R., Zatta P. Investigation of the aluminium binding in $\mathrm{Al}(\mathrm{III})$-treated neuroblastoma cells. J. Anal. Atom. Spectrom. $2004 ; 19$ : 41-45.

18. Baruthio F., Rieger B., Biette P., Pierre F. Spéciation de l'arsenic et l'urine humaine après exposition professionnelle. Ann. Toxicol. Anal. 2001 ; 13 : 186-95.

19. Okina M., Yoshida K., Kuroda K., Wanibuchi H., Fukushima S., Endo G. Determination of trivalent methylated arsenicals in rat urine by liquid chromatography-inductively coupled plasma mass spectrometry after solvent extraction. J. Chromatogr. B, 2004 ; 799 : 209-15.

20. Nam S.H., Kim J.J., Han S.S. Direct determination of total arsenic and arsenic species by ion chromatography coupled with inductively coupled plasma mass spectrometry. Bull. Korean Chem. Soc., 2003 ; 24 : 1805-8.

21. Van Hulle M., Zhang C., Schotte B., Mees L., Vanhaecke F., Vanholder R., Zhang X.R., Cornelis, R. Identification of some arsenic species in human urine and blood after ingestion of Chinese seaweed Laminaria. J. Anal. Atom. Spectrom. 2004 ; 19 : 58-64.

22. Shinohara A., Chiba M., Kondo M., Abou-Ahakra F.R., Walker H., Kobayashi K., Inaba Y. Speciation of arsenic compounds in human urine by HPLC/hexapole collision cell ICP-MS. Bunseki Kagaku, 2004 ; 53 : 589-93
23. B'Hymer C., Caruso J.A. Arsenic and its speciation analysis using high-performance liquid chromatography and inductively coupled plasma mass spectrometry. J. Chromatogr. A, 2004 ; 1045 : 1-13.

24. Lai V., Sun Y., Ting E., Cullen W., Reimer K. Arsenic speciation in human urine : Are we all the same? Toxicol. Appl. Pharmacol. 2004 ; 198 : 297-306.

25. Cairns W., Ebdon L., Hill S.A. High performance liquid chromatography - inductively coupled plasmamass spectrometry interface employing desolvation for speciation studies of platinum in chemotherapy drugs. Fresenius J. Anal. Chem. 1996 ; 355 : 202-8.

26. Hann S., Koellensperger G., Stefánka Zs., Stingeder G., Fürhacker M., Buchberger W., Mader R.M. Application of HPLC-ICP-MS to speciation of cisplatin and its degradation products in water containing different chloride concentrations and in human urine. J. Anal. Atom. Spectrom. $2003 ; 18: 1391-95$.

27. Bendahl L., Gammelgaard B. Separation and identification of Se-methylselenogalactosamine - A new metabolite in basal human urine - By HPLC-ICP-MS and CE-nanoESI-(MS). J. Anal. Atom. Spectrom. 2004 ; 19 : 950-7.

28. Gammelgaard B., Bendahl L. Selenium speciation in human urine samples by LC- and CE-ICP-MS Separation and identification of selenosugars. J. Anal. Atom. Spectrom. $2004 ; 19$ : 135-42.

29. Nyman D.W., Stratton M.S., Kopplin M.J., Dalkin B.L., Nagle R.B., Gandolfi A.J. Selenium and selenomethionine levels in prostate cancer patients. Cancer Detection and Prevention 2004 ; $28: 8-16$.

30. Miekeley N., Mortari S., Schubach A. Monitoring of total antimony and its species by ICP-MS and on-line ion chromatography in biological samples from patients treated for leishmaniasis. Anal. Bioanal. Chem. 2002 ; $372:$ 495-502.

31. Polec K., Garcia-Arribas O., Perez-Calvo M., Szpunar J., Ribas-Ozonas B., Lobinski R. Identification of cadmiumbioinduced ligands in rat liver using parallel HPLCICP-MS and HPLC-Electrospray MS. J. Anal. Atom. Spectrom. $2000 ; 15: 1363-8$.

32. Sun Y.C., Lin C.Y., Wu S.F., Chung Y.T. Evaluation of on-line desalter-inductively coupled plasma-mass spectrometry system for determination of $\mathrm{Cr}$ (III), $\mathrm{Cr}$ (VI), and total chromium concentrations in natural water and urine samples. Spectrochim. Acta Part B 2006 ; 61 : 230-4.

33. Quintana M., Klouda A.D., Ochsenkühn-Petropoulou M., Michalke B. Size characterization of manganese species from liver extracts using size exclusion chromatography inductively coupled plasma mass spectrometry. Anal. Chim. Acta 2005 ; 554 : 130-5.

34. Séby, F. Communication personnelle. 2006.

35. McSheehy S.,PohlP.,Lobinski R., Szpunar J.Investigation of arsenic speciation in oyster test reference material by multidimentional HPLC-ICP-MS and electrospray tandem mass spectrometry (ES-MS-MS). Analyst. 2001 ; $126: 1055-62$.

36. Garraud H., Vacchina V., Séby F., Dumont J., Sirot V., Guérin T., Leblanc J.C. Méthodologies analytiques pour la spéciation des métaux dans les produits de la mer dans le cadre d'une approche bénéfice/risque (étude CALIPSO). Ann. Toxicol. Anal. 2007 ; 19 : 71-80. 
37. Vacchina V., Torti L., Allievi C., Lobinski R. Sensitive species-specific monitoring of a new triplatinum anticancer drug and its potential related compounds in spiked human plasma by cation-exchange HPLC-ICP-MS. J. Anal. Atom. Spectrom. $2003 ; 18$ : 884-90.

38. Ruiz Encinar J., Schaumlöffel D., Ogra Y., Lobinski R. Determination of selenomethionine and selenocysteine in human serum using speciated isotope dilution-capillary HPLC-inductively coupled plasma collision cell mass spectrometry. Anal. Chem. 2004 ; 76 : 6635-42.

39. Leblanc J.C. CALIPSO - Etude des Consommations Alimentaires de produits de la mer et Imprégnation aux éléments traces, Polluants et Oméga 3. AFFSSA, Ministère de l'Agriculture et de la Pêche, INRA, 2006 ; 160pp.

40. McSheehy S., Dumont J., Garraud H., Donard O.F.X., Leblanc J.C., Sirot V., Christopher S., Davis W.C., Shaw P. Nash M. Methylmercury $\left(\mathrm{MeHg}^{+}\right)$and inorganic mercury $\left(\mathrm{Hg}^{2+}\right)$ determination in blood using GC-ICPMS. Winter Conference 2006, Tuckson (Arizona, USA), Janvier 2006.

41. Ballihaut G., Tastet L., Pecheyran C., Bouyssière B., Donard O., Grimaud R., Lobinski R. Biosynthesis, purification and analysis of selenomethionyl calmoduline by gel electrophoresis-laser ablation-ICP-MS and capillary HPLC-ICP-MS peptide mapping following ingel tryptic digestion. J. Anal. At. Spectrom. 2005 ; 20 : 493-99.

42. Lobinski R., Szpunar J. Biochemical speciation analysis by hyphenated techniques. Anal. Chimica Acta 1999 ; $400: 321-32$. 